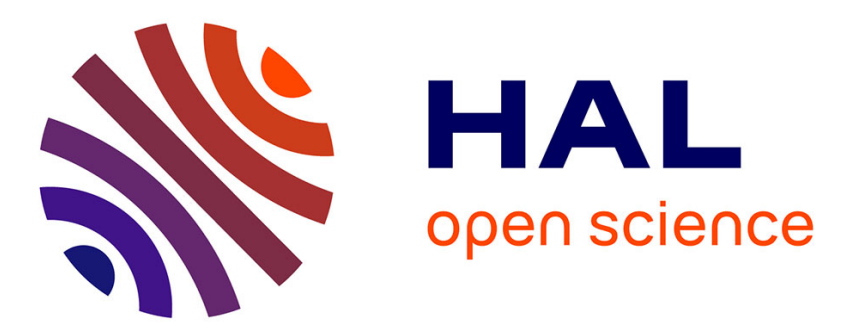

\title{
A power flow mode approach dedicated to structural interface dynamic characterization
}

Thomas Weisser, Emmanuel Foltête, Noureddine Bouhaddi, Luc-Olivier Gonidou

\section{- To cite this version:}

Thomas Weisser, Emmanuel Foltête, Noureddine Bouhaddi, Luc-Olivier Gonidou. A power flow mode approach dedicated to structural interface dynamic characterization. Journal of Sound and Vibration, 2015, 334, pp.202-218. 10.1016/j.jsv.2014.09.012 . hal-01511294

\author{
HAL Id: hal-01511294 \\ https://hal.science/hal-01511294
}

Submitted on 20 Apr 2017

HAL is a multi-disciplinary open access archive for the deposit and dissemination of scientific research documents, whether they are published or not. The documents may come from teaching and research institutions in France or abroad, or from public or private research centers.
L'archive ouverte pluridisciplinaire HAL, est destinée au dépôt et à la diffusion de documents scientifiques de niveau recherche, publiés ou non, émanant des établissements d'enseignement et de recherche français ou étrangers, des laboratoires publics ou privés.

\section{(c)(1)}

Distributed under a Creative Commons Attribution| 4.0 International License 


\title{
A power flow mode approach dedicated to structural interface dynamic characterization
}

\author{
Thomas Weisser ${ }^{\mathrm{a}, *}$, Emmanuel Foltête ${ }^{\mathrm{a}}$, Noureddine Bouhaddi ${ }^{\mathrm{a}}$, Luc-Olivier Gonidou ${ }^{\mathrm{b}}$ \\ ${ }^{a}$ Département Mécanique Appliquée, Institut FEMTO-ST, 24 chemin de l'Épitaphe, 25000 Besançon, France \\ ${ }^{b}$ Centre National d'Études Spatiales, Direction des Lanceurs, 52 rue Jacques Hillairet, 75612 Paris Cedex, France
}

\begin{abstract}
This study is related to structural vibration transmission and aims to propose a new approach to dynamically characterize the interface between different substructures of a complex coupled structure. A power flow mode method based on the imaginary part of the dynamic flexibility matrix is presented, which allows determining eigenvalues and eigenvectors representing respectively qualitative and quantitative information on the power flowing inside the structure. It is further applied to study the power transmitted at the interface, making it possible to identify the direction associated to the dominant power flow patterns and to quantify their contributions. Specific properties dealing with moment excitation and high-frequency approximation are also detailed to provide complementary insights into their dynamic meaning and behaviour.
\end{abstract}

Keywords: structural dynamics, vibration transfer, power flow, interface forces, substructuring

\section{Introduction}

\subsection{General background}

Complex mechanical structures usually encountered in the automotive or aerospace industries are composed of an assembly of several components, often exhibiting different mechanical properties and joined at their interfaces by different junction types. The various dynamic behaviours of these substructures and the applied external dynamic loadings may generate important forces on the main structure, resulting in high acceleration responses of the onboard equipment, potentially affecting adversely their performance, reliability and security. It is therefore necessary to protect these components from these harsh interface loadings by isolating them from the rest of the structure.

Lots of researches have been performed during the last four decades to analyze, to model and to control these vibration transmissions. Particular attention has been spent on structure-borne sound, which is defined as the vibratory energy associated to elastic waves flowing through solid media. Initially concerning vibroacoustic issues of radiated noise reduction, this approach still remains relevant for structural vibration isolation, resulting from the internal forces acting at the interface between a source and receiver substructure. However, the derived methodologies are generally based on simplifying hypotheses which tend to be unverified when dealing with complex structures: no rigid-like receiver substructure, complex interface topologies, multiple junctions,... The whole vibration transfer phenomena may also be hardly understood by independently comparing the interface force and velocity vectors $[1,2]$.

Refined isolation methodologies have been proposed, based on the power flow dissipated or transmitted between the different substructures, providing new quantities of interest which allow characterizing the receiver or source substructures, and possibly the junction components. Even if their final purpose is to reduce vibration response levels, these methods also aim to bring new insights on vibration exchanges, such as the nature of the different couplings or the principal transmission paths.

*thomas.weisser@univ-fcomte.fr 


\subsection{Dynamic isolation of coupled structures}

In their reference book [3], Cremer et al. noticed that vibration transfer in a complex coupled structure can be divided into three main stages: a physical mechanism first generates oscillations, whose associated energy is thus transmitted to the structure and finally allocated to the different components. This process is usually handled by distinguishing transfers between a source substructure and a receiver substructure, through junction components, whose definitions depend on the adopted goal. The vibration isolation problem then comes down to minimizing vibrations transmitted to the receiver [4]. This is generally performed by decoupling the structural resonances or the dynamic response levels of the source and receiver substructures. Hence it is necessary to link their different input parameters, such as design variables, to the applied structural modifications (mass, stiffness and/or damping distributions) or the computed isolation efficiency metrics.

As emphasized above, regarding the initial subdivision of the vibration transfer problem, interface variables are of key interest. Generally, the dynamic behaviour at the interface is described using velocities and forces. These primal vector quantities, complex valued and directly available experimentally or numerically, enable a detailed spatial description, for example by using local transfer functions. However, power flow computations based on quadratic real variables, resulting from space and/or time/frequency averages, seem to be particularly relevant and efficient $[5,1]$.

\subsection{Power flow analysis}

The first ideas associated to power flow analysis have been published by Lyon and Maidanik concerning vibration propagation in simple systems [6]. The related basic concepts have been defined by Goyder and White, who proposed a comprehensive theoretical study of structural power flow in flexible mono- and bi-dimensional structures [7, 8, 9]. This also corresponds to the effective power which can be derived, in harmonic state, from the real part of the complex power [3]. The authors demonstrated that power flow constitutes a single parameter allowing to quantify and to compare, in an efficient way, the main effects associated to structural vibrations. They have especially been focusing on rigid source substructures joined to flexible receiver ones by spring elements. Pinnington and White also used this approach to study the impact of infinite structure hypothesis on average power flow estimates [10]. Pinnington then proposed a method based on the envelope of frequency response functions, experimentally obtained, to study the power flow absorbed by a finite structure: this can be expressed as the sum of the power flows introduced by the different measured vibration modes, which are associated to particular configuration of generalized forces [11]. This global power flow analysis approach can be strengthened by taking into account the local properties of the structure using mobility based methods [12]. In the case of complex assembled structures, deriving these quantities at each interface point (including cross terms) allows to fully determine the total transmitted power flow [13, 14].

However such raw data make it difficult to properly describe the vibration transmission mechanisms. Hence Mondot and Petersson introduced two new quantities to fully characterize a source substructure and the transmitted power flow [15]: the source descriptor representing its ability to deliver power, and the coupling function denoting the proportion of power flow transmitted to the receiver. These concepts have been extended to multiple connection points using effective [16] or interface mobilities. The vibration source can therefore be decomposed into multiple orders of a series, associated to the dynamic behaviour of the interface (rigid body or elastic motions,...) $[17,18]$.

Focusing on a qualitative description of the transmitted power, and based on the equality between the timeaveraged power of a system and the energy dissipated by its damping, Miller et al. first proposed to perform an eigenvalue analysis of a power matrix at the interface, to determine frequency ranges and mode combinations which cause the junction to dissipate power [19]. The same idea can be found in the multipole method developed by Pinnington, which describes the power generated by a source as flowing through a set of vibration poles, constituting a spatial vectorial basis of the size of the interface degrees-of-freedom $(D O F s)$. By coupling this approach to frequency synthesis ones, Su et al. proposed a mobility-based power flow mode method. It consists in transforming a set of source forces or velocities into a new set of power flows: these are associated to modal forces or velocities weightened by orthogonal functions derived from the eigenproblem of a mobility matrix [20]. The power transmitted from a source to a receiver substructure by a set of forces can thus be seen as being injected by so many independent power flow modes. To overcome the strong dependency of these modes on the physical parameters of the system and on the frequency, Xiong et al. introduced a damping-based power flow mode theory, relying exclusively on damping parameters [21]. The authors formulated power flow design theorems to passively and actively control some required energy flow dissipation levels and patterns. Finally, other works have been exposed by Bessac et al. to characterize 
vibration transmission between two substructures joined by pure stiffness junctions. Based on the eigenproblem of a dimensionless coupling matrix, this method provides the coupling intensity and the associated prevailing transmission paths [22].

\subsection{Adopted approach}

The method proposed in this paper is dedicated to low frequency vibrations of complex structures. While standard modal approaches actually allow deriving efficient isolation results (e.g. by shifting the natural frequencies of the receiver, reducing the number of significant responding modes, or "nodalizing" the structure by modifying spatial couplings and responses between components), such approaches do not allow to characterize the vibration transfer mechanisms at the interface between different substructures.

A power flow mode approach, as initially proposed by $\mathrm{Su}$ et al. [20], has been retained to combine the advantages of both finite element analysis, i.e. fine spatial description, complex geometry handling, vector quantities, and power flow analysis, i.e. quadratic variables and scalar characteristic quantities. However, this method is based on a displacement formulation and aims to derive power flow modes to qualitatively and quantitatively characterize interface forces in order to estimate their ability to transmit power flow between components [23].

Following this introduction, the basic theoretical formulation of the proposed method is developed in section 2 to introduce the concept of power flow mode. A simple parametric study is proposed to emphasize the complex dynamic behaviour of these quantities. Section 3 presents the extension of this approach to characterize the interface forces between two substructures, leading to the notion of power flow dominant subspace. Finally, the whole method is applied to an academic coupled beam structure and some special features of the power flow modes are enlightened, before ending with some general conclusions and perspectives.

\section{Theoretical formulation}

\subsection{A flexibility-based approach}

To illustrate this basic formulation, a classical two-DOFs spring-mass-damper system is introduced figure 1 whose initial parameters are given table 1 .

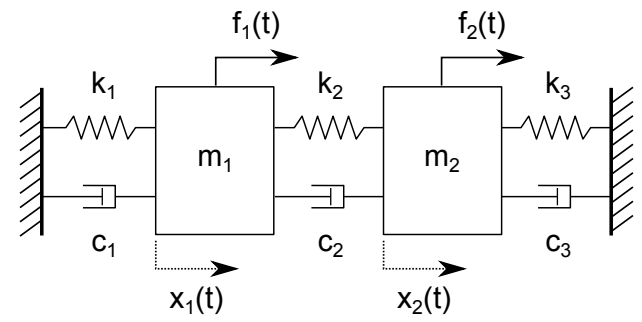

Figure 1: Two-DOFs spring-mass-damper system

\begin{tabular}{ccc}
\hline Mass & $(k g)$ & $m_{1}=6, m_{2}=4$ \\
\hline Stiffness & $(N / m)$ & $k_{1}=2000, k_{2}=8000, k_{3}=6000$ \\
\hline Damping & $(N s / m)$ & $c_{1}=6, c_{2}=8, c_{3}=2$ \\
\hline
\end{tabular}

Table 1: Description of the two-DOFs system

As thoroughly detailed in the work of Bobrovnitskii [24] the average power flow of a $N$-DOFs system, at a given frequency $\omega$, can be derived from the real part of the complex power

$$
P_{0}(\omega)=\mathfrak{R e}\left\{P^{*}(\omega)\right\}=\frac{1}{2} \mathfrak{R e}\left\{\mathbf{v}^{H} \mathbf{f}\right\}=\frac{1}{2} \mathfrak{R e}\left\{\mathbf{f}^{H} \mathbf{v}\right\}
$$


where $\mathbf{f} \in \mathbb{C}^{(N, 1)}$ and $\mathbf{v} \in \mathbb{C}^{(N, 1)}$ denote the force and velocity complex amplitudes, respectively ${ }^{1}$. This can be rewritten as

$$
P_{0}(\omega)=\frac{1}{4}\left(\mathbf{f}^{H} \mathbf{v}+\mathbf{v}^{H} \mathbf{f}\right)
$$

Noticing that $\mathbf{v}=j \omega \mathbf{x}$ in harmonic state, where $\mathbf{x} \in \mathbb{C}^{(N, 1)}$ is the displacement vector, it becomes

$$
P_{0}(\omega)=\frac{j \omega}{4}\left(\mathbf{f}^{H} \mathbf{x}+\mathbf{x}^{H} \mathbf{f}\right)
$$

In order to express $P_{0}$ as a function of the parameters of the system, the dynamic flexibility matrix $\boldsymbol{\Gamma} \in \mathbb{C}^{(N, N)}$ is introduced

$$
\boldsymbol{\Gamma}(\omega)=\left(-\omega^{2} \mathbf{M}+j \omega \mathbf{C}+\mathbf{K}\right)^{-1}
$$

where $\mathbf{M} \in \mathbb{R}^{(N, N)}, \mathbf{C} \in \mathbb{R}^{(N, N)}$ and $\mathbf{K} \in \mathbb{R}^{(N, N)}$ denote the mass (symmetric and positive definite), damping (symmetric and semi-positive definite) and stiffness (symmetric and semi-positive definite) matrices of the structure, respectively. By substituting it into the previous equation

$$
P_{0}(\omega)=\frac{j \omega}{4} \mathbf{f}^{H}\left[\boldsymbol{\Gamma}(\omega)-\boldsymbol{\Gamma}(\omega)^{H}\right] \mathbf{f}
$$

The average power can then be expressed as a quadratic form associated to the imaginary part of the dynamic flexibility matrix

$$
P_{0}(\omega)=-\frac{\omega}{2} \mathbf{f}^{H} \mathfrak{J m}\{\boldsymbol{\Gamma}(\omega)\} \mathbf{f}
$$

Considering that the average power remains greater or equal to zero and that $\mathfrak{I m}\{\boldsymbol{\Gamma}(\omega)\}$ is a real symmetric matrix, it can be derived that it is also non-positive definite.

The main idea is now to optimize this power with regard to the external applied forces [25]. A discretized Rayleigh-like quotient is derived by weightening equation (6) by the squared norm of these forces

$$
\mathcal{R}(\omega)=-\frac{\omega}{2} \frac{\mathbf{f}^{H} \mathfrak{J m}\{\boldsymbol{\Gamma}(\omega)\} \mathbf{f}}{\mathbf{f}^{H} \mathbf{f}}
$$

Optimizing $\mathcal{R}$ at a given frequency $\omega=\omega_{0}$ amounts to differentiating this equation with respect to the external forces and, according to the stationarity property of the quotient ${ }^{2}$, leads to solve the following equivalent eigenproblem

$$
\left[\mathfrak{J m}\left\{\boldsymbol{\Gamma}\left(\omega_{0}\right)\right\}-s_{v} \mathbf{I}_{N}\right] \mathbf{g}_{v}=0, \quad v=1, \ldots, N
$$

where $\mathbf{I}_{N}$ denotes the identity matrix of size $N . \mathbf{S}=\operatorname{diag}\left(s_{v}\right) \in \mathbb{R}^{(N, N)}$ is defined as a non-positive definite diagonal matrix containing the power flow eigenvalues arranged in ascending order

$$
s_{1} \leq s_{2} \leq \cdots \leq s_{n} \leq 0
$$

and $\mathbf{G}=\left[\begin{array}{lll}\mathbf{g}_{1} \ldots \mathbf{g}_{N}\end{array}\right] \in \mathbb{R}^{(N, N)}$ represents the orthogonal matrix of the normalized power flow eigenvectors. These matrices both satisfy the following orthogonal relations

$$
\begin{gathered}
\mathbf{G}^{T} \mathbf{G}=\mathbf{I}_{N} \\
\mathbf{G}^{T} \mathfrak{J} \mathrm{m}\left\{\boldsymbol{\Gamma}\left(\omega_{0}\right)\right\} \mathbf{G}=\mathbf{S}
\end{gathered}
$$

These power flow modes are independent of the applied external forces and allow to precisely characterize, at a given frequency $\omega=\omega_{0}$, the average power flow potentially available in the structure

\footnotetext{
$1 .{ }^{H}$ is the complex transpose conjugate operator

${ }^{2}$ This result directly derives from the properties of symmetric matrices, as detailed in [26].
} 
- the eigenvalues give quantitative information about the amplitude of the power flow associated to each mode ;

- the eigenvectors (or eigenforces) give qualitative information, representing paths of principal directions transmitting the respective amount of power flow of the associated eigenvalues.

It must be noticed that such equivalent eigenproblem must be solved at each considered frequency: although mathematically independent, the derived power flow modes correspond to physical quantities whose behaviour is assumed to be continuous. A MAC-based mode pairing procedure has thus been implemented to overcome this difficulty ( $c f$. section 2.3).

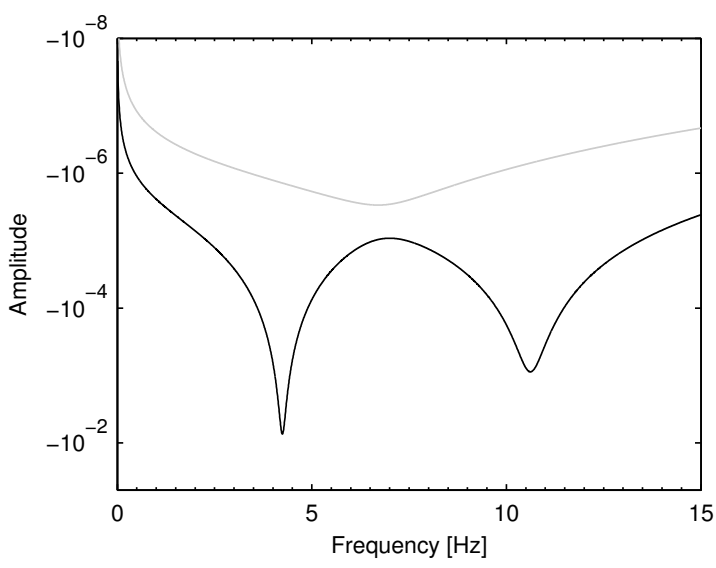

(a) Eigenvalues: $-s_{1},-s_{2}$

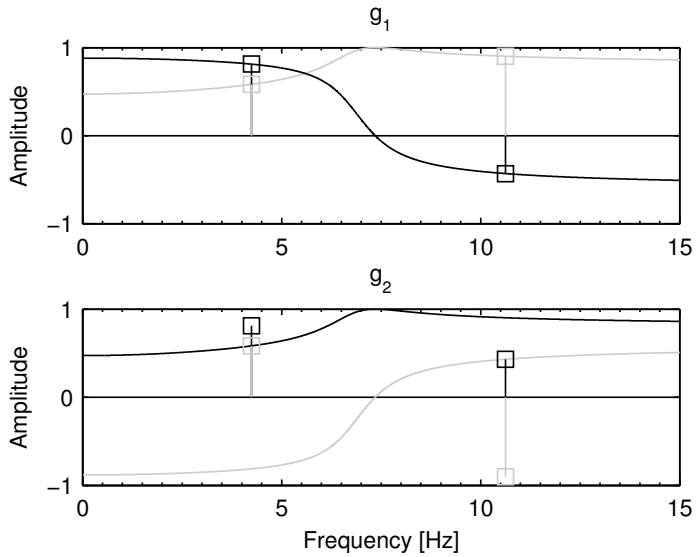

(b) Eigenforces: $-D O F_{1},-D O F_{2}$ $\square$ normal vibration modes

Figure 2: Power flow modes of the 2-DOFs discrete system

Figure 2 (a) displays the eigenvalues versus frequency of the two-DOFs discrete system: these are all negative and tend toward zero at zero frequency. The first eigenvalue $s_{1}$ has amplitude resonances located close to natural vibration frequencies. It can also be seen that $\left|s_{1}\right| \gg\left|s_{2}\right|$, except in the antiresonance zone where both eigenvalues have the same order of magnitude: $s_{2}$ then reaches its maximum. Figure 2 (b) represents the evolution of each component of the eigenforces versus frequency. These vectors are rotating and almost exchanging their initial deformed shapes around the antiresonance. However, at resonance frequencies, the eigenforce associated to the dominant eigenvalue $s_{1}$ is collinear to the normal vibration modes, verifying the modal appropriation principle [27]. Furthermore, the second eigenvector of the power flow mode basis is thus orthogonal, representing the directions injecting the least power flow to the structure.

\subsection{Parametric study of power flow modes}

A brief study has been performed to observe the influence of the physical parameters of the system on the frequency behaviour of the power flow modes. To further emphasize the link between the physical parameters of the system and its power flow modes, an analytical expression of the power flow eigenvalues is also determined in a particular simplified case to introduce curve veering and mode-mixing phenomena.

\subsubsection{Influence of the physcial parameters}

It has been noticed that a variation of mass or stiffness parameters results in a frequency shift of the power flow resonances, similarly to natural vibration frequencies (e.g. figure $3(\mathrm{a}),[-17 \% ;+11 \%]$ for $s_{1}$ and $[-6 \% ;+6 \%]$ for $\left.s_{2}\right)$. This variation also controls the amount of shape exchange between the eigenforces, whose rotation speed is illustrated by the slope of the different curves around their inflection point, figure 3 (b). Assuming a proportional (or Rayleigh) 
damping of the system, i.e. $\mathbf{C}=a \mathbf{K}+b \mathbf{M}$, it has been observed that a modification of parameter $a$ acts on both resonances: classically, as the damping increases, the bandwidth widens and the amplitude peak decreases, figure 4 (a). Moreover, it can be noticed that even if there is a frequency shift of the antiresonance, the gap between both eigenvalues remains constant, as confirmed by the constant slope of the different component curves, figure 4 (b).

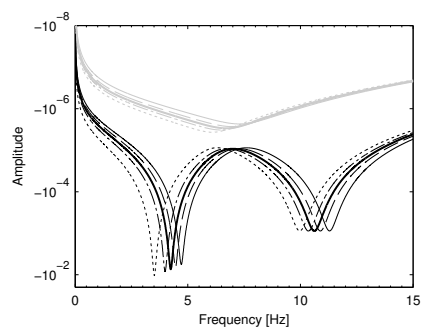

(a) Eigenvalues: $-s_{1},-s_{2}$

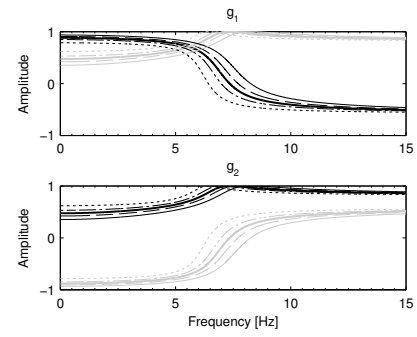

(b) Eigenforces: $-D O F_{1},-D O F_{2}$

Figure 3: Influence of a stiffness variation of $k_{3}$ :

- initial, $\cdots-50 \%,-\cdot--20 \%,--+20 \%,-+50 \%$

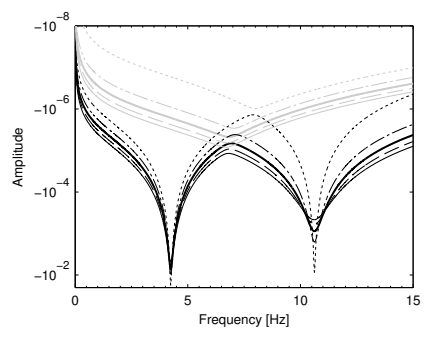

(a) Eigenvalues: $-s_{1},-s_{2}$

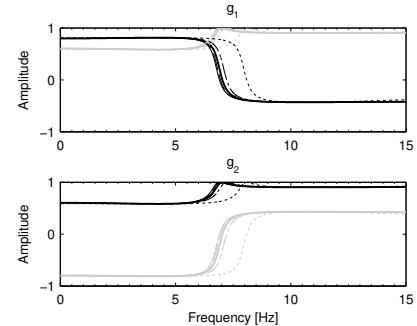

(b) Eigenforces: $-D O F_{1},-D O F_{2}$

Figure 4: Influence of a damping variation of $a$ :

- initial, $\cdots-100 \%,-\cdot--50 \%,--+50 \%,-+100 \%$

This parametric study highlights a complex behaviour of the power flow eigenvalues and eigenforces, although closely related to the normal vibration modes of the system. An analytical expression of the eigenvalues is thus derived in the next section to clarify the connection with modal characteristics.

\subsubsection{Simplified analytical insight}

To avoid oversized complicated expressions due to multiple parameters, the following assumptions are made

$$
\left\{\begin{array}{l}
m_{1}=m_{2}=m \\
k_{1}=k_{3}=k_{I} \quad \text { and } \quad k_{2}=k_{I I} \\
c_{1}=c_{3}=c_{I} \quad \text { and } \quad c_{2}=c_{I I}
\end{array}\right.
$$

The associated dynamic equilibrium equations verify Caughey's condition, i.e. proportional damping assumption,leading to real normal vibration modes. A modal superposition approach is adopted to conveniently derive the imaginary part of the dynamic flexibility matrix, and the power flow eigenvalues are finally obtained by solving equation 8

$$
\begin{gathered}
s_{1}(\omega)=2 \gamma_{1}(\omega)=-\frac{\omega c_{I}}{\left(k_{I}-\omega^{2} m\right)^{2}+\left(\omega c_{I}\right)^{2}}<0 \\
s_{2}(\omega)=2 \gamma_{2}(\omega)=-\frac{\omega\left(c_{I}+2 c_{I I}\right)}{\left(k_{I}+2 k_{I I}-\omega^{2} m\right)^{2}+\left(\omega\left(c_{I}+2 c_{I I}\right)\right)}<0
\end{gathered}
$$


It can be seen that each denominator is identical to the usual frequency response function one, confirming the observed influence of the different system parameters. These power flow modes are represented figure 5. However in this particular case, each eigenvalue admits a unique resonance and the antiresonance is replaced by a crossing corresponding to a double-order multiple eigenvalue. This is emphasized by the fact that only equation (14) depends on the coupling parameters $\left(k_{I I}, c_{I I}\right)$ and is thus associated to the second (out of phase) normal vibration mode (figure 5 (b)).

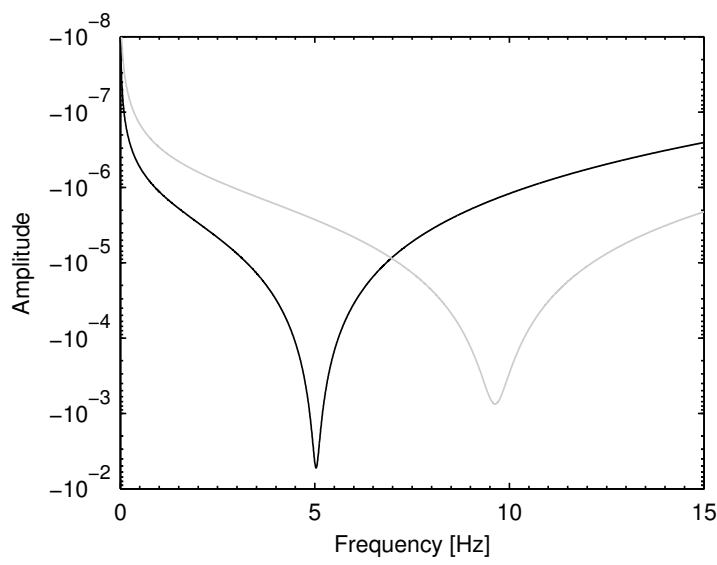

(a) Eigenvalues: $-s_{1},-s_{2}$
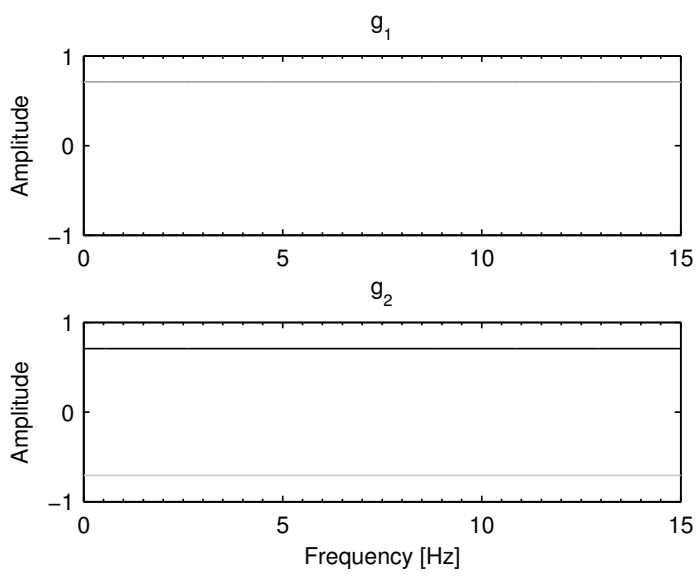

(b) Eigenforces: $-D O F_{1},-D O F_{2}$

Figure 5: Particular case of power flow modes

In order to determine whether the power flow resonance frequencies correspond to the normal vibration ones, these are determined by canceling the derivatives of $s_{1}$ and $s_{2}$ with regard to $\omega$

$$
\begin{aligned}
\omega_{s_{1} \text { max }}^{2}=\frac{1}{6 m^{2}}\left(2 m k_{I}-c_{I}^{2}+\sqrt{16 m^{2} k_{I}^{2}-4 m k_{I} c_{I}^{2}+c_{I}^{4}}\right) \\
\omega_{s_{2} \text { max }}^{2}=\frac{1}{6 m^{2}}\left(2 m\left(k_{I}+2 k_{I I}\right)-\left(c_{I}+2 c_{I I}\right)^{2}+\right. \\
\left.\sqrt{16 m^{2}\left(k_{I}+2 k_{I I}\right)^{2}-4 m\left(k_{I}+2 k_{I I}\right)\left(c_{I}+2 c_{I I}\right)^{2}+\left(c_{I}+2 c_{I I}\right)^{4}}\right)
\end{aligned}
$$

Although different, these values tend toward vibration frequencies as damping tends toward zero

$$
\lim _{\substack{c_{I} \rightarrow 0 \\ c_{I I} \rightarrow 0}} \omega_{s_{1} \max }^{2}=\frac{k}{m}=\omega_{1}^{2} \quad \text { and } \quad \lim _{\substack{c_{I} \rightarrow 0 \\ c_{I I} \rightarrow 0}} \omega_{s_{2} \max }^{2}=\frac{k+2 k_{j}}{m}=\omega_{2}^{2}
$$

Finally, as each eigenvalue is associated to a normal vibration mode of the system, it can be noticed that the eigenforces displayed figure 5 (b) are frequency-independent and collinear to each vibration mode.

\subsection{Curve veering and mode-mixing phenomena}

Even if power flow modes exhibit particular modal-influenced behaviours in the case of a symmetrical distribution of the system physical parameters, it has also been noticed that the slightest perturbation leads to one (or more) dominant power flow modes with complex frequency behaviours.

This kind of phenomenon has already been enlightened for multiple-order normal vibration modes [28, 29]: figure 6 shows that small perturbations can generate interactions, sometime in a very localized way, between the eigenvalues 


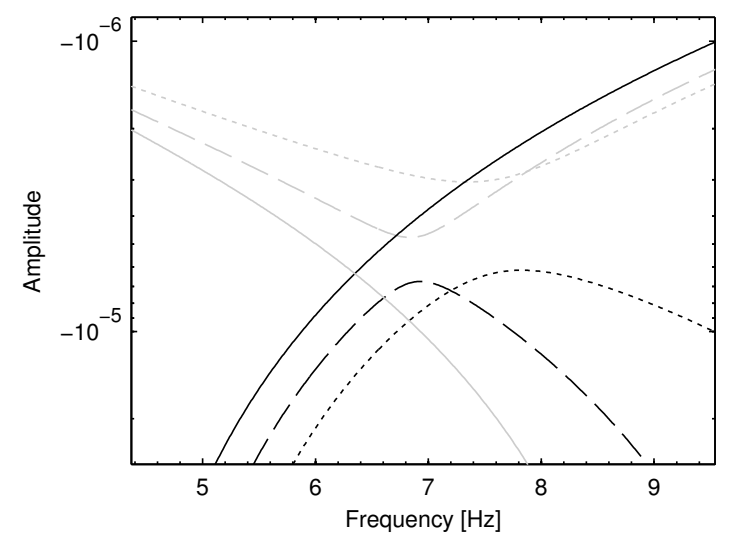

Figure 6: Loci veering of power flow eigenvalues

$-s_{1},-s_{2} ; \cdots 3 m_{2}=m_{1},--3 m_{2}=2 m_{1},-m_{2}=m_{1}$

whose loci will come close until they reach a minimal distance and then diverge. This loci veering effect also implies a mode-mixing phenomenon of the associated eigenforces, illustrated by a quick but continuous exchange of their deformed shapes. Figures 7 (a) and (b) represent a simple criterion defined by

$$
\chi_{i j}^{(k, l)}=\frac{\left|\mathbf{g}_{k}^{\left(\omega_{i}\right)}{ }^{T} \mathbf{g}_{l}^{\left(\omega_{j}\right)}\right|^{2}}{\left\|\mathbf{g}_{k}^{\left(\omega_{i}\right)}\right\|^{2}\left\|\mathbf{g}_{l}^{\left(\omega_{j}\right)}\right\|^{2}}
$$

applied to the eigenforces associated to the eigenvalues of figure 6 ( $k$ and $l$ represent the compared power flow modes and $\omega_{i}$ and $\omega_{j}$ the considered frequencies). At each frequency, orthogonality or collinearity of the eigneforces result in a deep blue or deep red color, respectively: mode-mixing is thus illustrated by intermediate colors, giving information on the quickness and the level of the veering phenomenon.

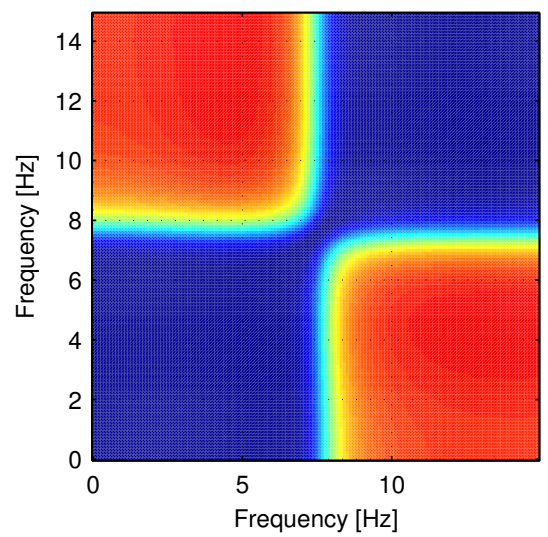

(a) $3 m_{2}=m_{1}$
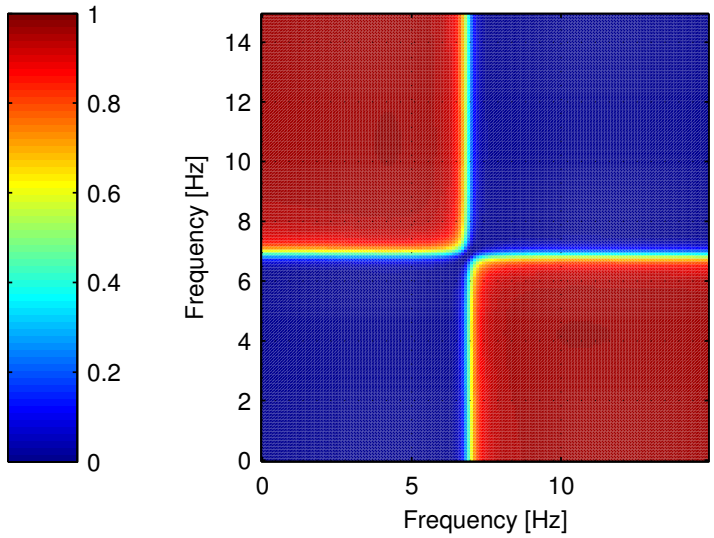

(b) $3 m_{2}=2 m_{1}$

Figure 7: Eigenforces mode-mixing criterion 


\section{Structural interface characterization}

The aim of this section is to apply the power flow mode method to characterize the interface forces between two components of an academic coupled structure depicted figure 8: a source substructure $(S)$ submitted to an external loading $\mathbf{f}^{(S)}$ and a passive receiver substructure $(R)$. The dynamic equilibrium equation of this structure is given by

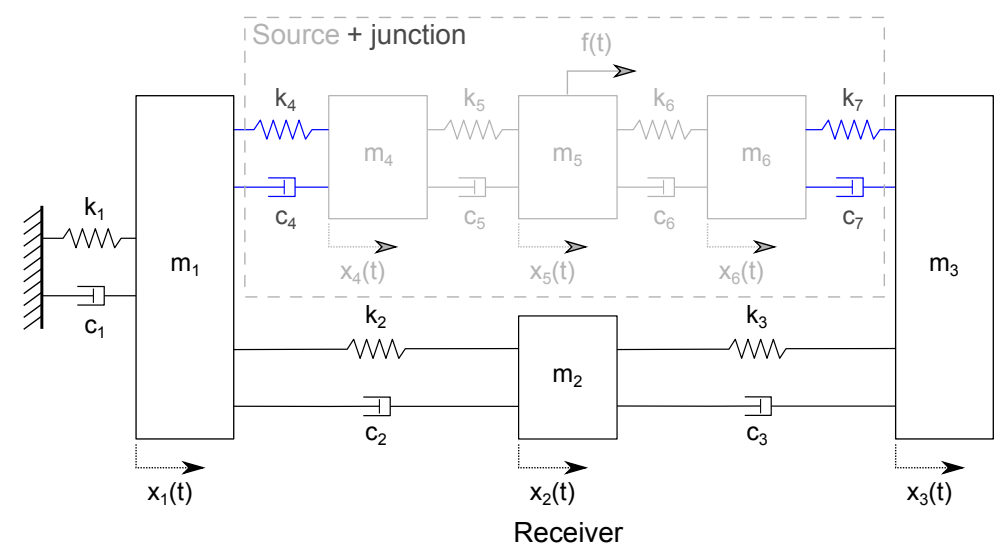

Figure 8: Six-DOFs spring-mass-damper coupled

$$
\left[\begin{array}{ccc}
\mathbf{Z}_{i i}^{(S)} & \mathbf{Z}_{i j}^{(S)} & \mathbf{0} \\
\mathbf{Z}_{j i}^{(S)} & \mathbf{Z}_{j j}^{(S)}+\mathbf{Z}_{j j}^{(R)} & \mathbf{Z}_{j i}^{(R)} \\
\mathbf{0} & \mathbf{Z}_{j i}^{(R)} & \mathbf{Z}_{i i}^{(R)}
\end{array}\right]\left(\begin{array}{c}
\mathbf{x}_{i}^{(S)} \\
\mathbf{x}_{j} \\
\mathbf{x}_{i}^{(R)}
\end{array}\right)=\left(\begin{array}{c}
\mathbf{f}_{i}^{(S)} \\
\mathbf{0} \\
\mathbf{0}
\end{array}\right)
$$

where subscripts $i$ and $j$ respectively denote internal and interfac DOFs, $\mathbf{Z}^{(\cdot)}$ is the frequency dependent dynamics stiffness matrix of a substructure and $\mathbf{x}^{(.)}$its dynamic response vector.

\subsection{Associated power flow modes}

\subsubsection{Substructuring approach}

To determine the power flow modes associated to the interface DOFs, the power transmitted between both substructures must be expressed with regard to the interface variables

$$
P_{j}^{t r}\left(\omega_{0}\right)=\frac{1}{2} \Re \mathrm{e}\left\{\mathbf{f}_{j}^{H} \mathbf{v}_{j}\right\}=\frac{j \omega}{4}\left(\mathbf{f}_{j}^{H} \mathbf{x}_{j}+\mathbf{x}_{j}^{H} \mathbf{f}_{j}\right)
$$

By decoupling equation (19) and considering the following compatibility equations

$$
\left\{\begin{array}{l}
\mathbf{x}_{j}^{(S)}=\mathbf{x}_{j}^{(R)}=\mathbf{x}_{j} \\
-\mathbf{f}_{j}^{(S)}=\mathbf{f}_{j}^{(R)}=\mathbf{f}_{j}
\end{array}\right.
$$

the equilibrium of the coupled structure can be classically reduced to

$$
\left[\mathbf{Z}_{j j}^{(R)}-\mathbf{Z}_{j i}^{(R)}\left[\mathbf{Z}_{i i}^{(R)}\right]^{-1} \mathbf{Z}_{i j}^{(R)}\right] \mathbf{x}_{j}=\mathbf{f}_{j}
$$

An expression of $\mathbf{x}_{j}$ can be further obtained by inverting this equation. However, it can also be verified using blocmatrix computation that this expression corresponds to the dynamic flexibility matrix of the receiver substructure, restricted to its interface $D O F S$

$$
\boldsymbol{\Gamma}_{j j}^{(R)}=\left[\mathbf{Z}_{j j}^{(R)}-\mathbf{Z}_{j i}^{(R)}\left[\mathbf{Z}_{i i}^{(R)}\right]^{-1} \mathbf{Z}_{i j}^{(R)}\right]^{-1}
$$

Equation (20) can finally be expressed as a quadratic form

$$
P_{j}^{t r}\left(\omega_{0}\right)=-\frac{\omega_{0}}{2} \mathbf{f}_{j}^{H} \mathfrak{J m}\left\{\boldsymbol{\Gamma}_{j j}^{(R)}\left(\omega_{0}\right)\right\} \mathbf{f}_{j}
$$




\subsubsection{Equivalent eigenproblem}

Similarly to section 2.1, minimizing equation (24) at each considered frequency amounts to solving the following equivalent eigenproblem

$$
\left[\mathfrak{I m}\left\{\boldsymbol{\Gamma}_{j j}^{(R)}\left(\omega_{0}\right)\right\}-s_{v} \mathbf{I}_{N_{j}}\right] \mathbf{g}_{v}=0, \quad v=1, \ldots, N_{j}
$$

where $N_{j}$ denotes the number of interface $D O F s^{3}$. The derived power flow modes also verify orthogonality relations

$$
\begin{gathered}
\mathbf{G}^{T} \mathbf{G}=\mathbf{I}_{N_{j}} \\
\mathbf{G}^{T} \boldsymbol{\Gamma}^{\mathfrak{J}} \mathbf{G}=\mathbf{S}
\end{gathered}
$$

where $\mathbf{G}=\left[\begin{array}{lll}\mathbf{g}_{1} & \ldots & \mathbf{g}_{N_{j}}\end{array}\right] \in \mathbb{R}^{\left(N_{j}, N_{j}\right)}$ and $\mathbf{S}=\operatorname{diag}\left(s_{v}\right) \in \mathbb{R}^{\left(N_{j}, N_{j}\right)}$.

It must be noticed that equation 25 only depends on the physical parameters of the receiver and the frequency: the derived power flow modes are independent of the interface forces applied by the source substructure to the receiver one, thus allowing to characterize the power potentially transmitted at the interface. At each considered frequency, the eigenforces represent the prevailing directions or paths through which the power proportional to the associated eigenvalues will flow.

By comparison with figure 2, figure 9 displays the power flow modes associated to the two interface DOFs of the six-DOFs discrete system. It can be observed that $\left|s_{1}\right| \gg\left|s_{2}\right|$, admitting power flow resonances at the normal vibration frequencies of the uncoupled receiver substructure (at 2.45, 5.55 and $13.35 \mathrm{~Hz}$ ). Moreover, figure 9 (b) shows that, at this particular frequencies, $\mathbf{g}_{1}$ is collinear to the normal vibration mode, restricted to the interface DOFs.

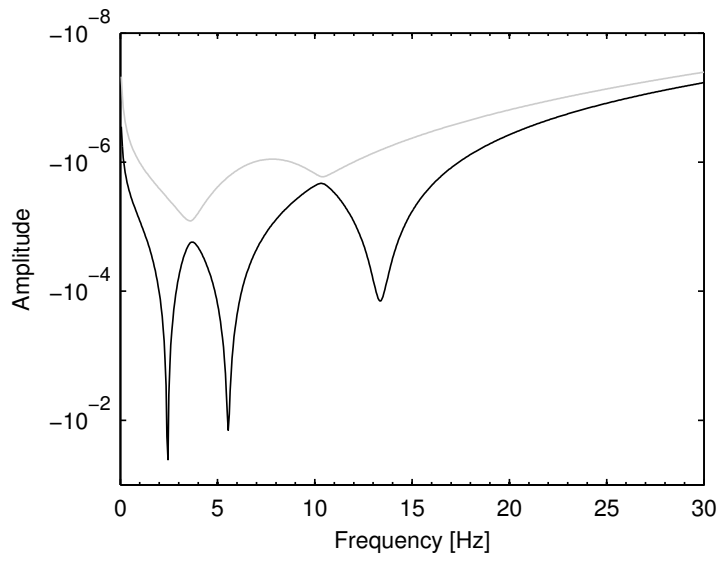

(a) Eigenvalues: $-s_{1},-s_{2}$
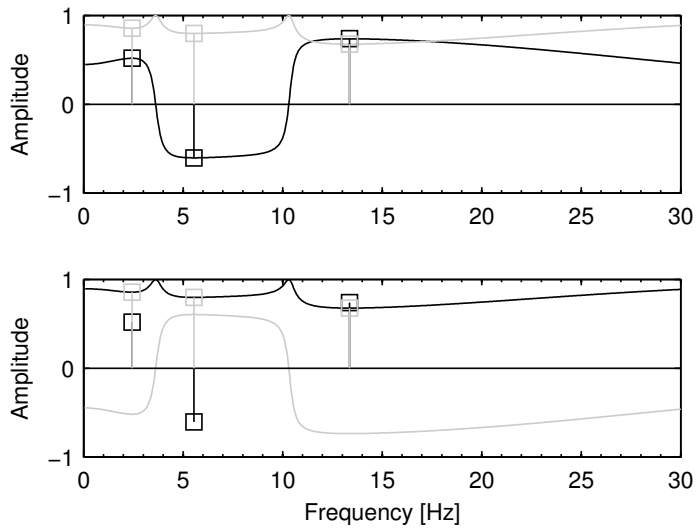

(b) Eigenforces: $-D O F_{1},-D O F_{2}$ $\square$ normal vibration modes

Figure 9: Power flow modes of the 6-DOFs discrete system

\subsection{Interface force decomposition}

The matrix $\mathbf{G}$ of the normalized eigenvectors $g_{v}$ derived equation (25) constitutes a full basis of size $N_{j}$ : these eigenforces span, at each considered frequency, the whole power flow pattern space associated to the interface. Hence it is possible to decompose the actual interface forces $\mathbf{f}_{j} \in \mathbb{C}^{\left(N_{j}, 1\right)}$ as a linear combination

$$
\mathbf{f}_{j}=\mathbf{G} \boldsymbol{\alpha}=\sum_{v=1}^{N_{j}} \alpha_{v} \mathbf{g}_{v}
$$

\footnotetext{
${ }^{3}$ To ease further readability, the following notation is adopted: $\boldsymbol{\Gamma}^{\mathfrak{J}}=\mathfrak{J m}\left\{\boldsymbol{\Gamma}_{j j}^{(R)}\right\}$.
} 
where $\boldsymbol{\alpha} \in \mathbb{C}^{\left(N_{j}, 1\right)}$ is a vector of complex-valued coefficients $\alpha_{v}=\alpha_{v}^{\mathfrak{R}}+j \alpha_{v}^{\mathfrak{J}}$ representing the participation of each eigenforce $\mathbf{g}_{v}$ in $\mathbf{f}_{j}$. These are easily determined using the orthogonal property of the basis given equation (27)

$$
\mathbf{g}_{\sigma}^{T} \mathbf{f}_{j}=\sum_{v=1}^{N_{j}} \alpha_{\nu} \mathbf{g}_{\sigma}^{T} \mathbf{g}_{v}=\alpha_{\sigma}
$$

By substituting this relation into equation (24)

$$
\begin{aligned}
P_{j}^{t r}\left(\omega_{0}\right) & =-\frac{\omega_{0}}{2} \mathbf{f}_{j}^{H} \Gamma^{\mathfrak{I}}\left(\omega_{0}\right) \mathbf{f}_{j}=-\frac{\omega_{0}}{2}\left(\left(\mathbf{G}^{T} \mathbf{f}_{j}^{\mathfrak{R}}\right)^{T} \mathbf{S}\left(\mathbf{G}^{T} \mathbf{f}_{j}^{\mathfrak{R}}\right)+\left(\mathbf{G}^{T} \mathbf{f}_{j}^{\mathfrak{J}}\right)^{T} \mathbf{S}\left(\mathbf{G}^{T} \mathbf{f}_{j}^{\mathfrak{J}}\right)\right) \\
& =-\frac{\omega_{0}}{2} \sum_{\nu=1}^{N_{j}}\left(\alpha_{v}^{\mathfrak{R}}\right)^{2} s_{v}+\left(\alpha_{v}^{\mathfrak{J}}\right)^{2} s_{\nu}=-\frac{\omega_{0}}{2} \sum_{\nu=1}^{N_{j}}\left|\alpha_{\nu}\right|^{2} s_{v}>0
\end{aligned}
$$

the power transmitted at the interface given equation (20) can be interpreted as the sum of the power independently transmitted by each of the $N_{j}$ power flow modes, also referred to as modal power flow $P_{v}$

$$
P_{j}^{t r}\left(\omega_{0}\right)=\sum_{\nu=1}^{N_{j}} P_{\nu}\left(\omega_{0}\right), \quad \text { with: } \quad P_{\nu}\left(\omega_{0}\right)=-\frac{\omega_{0}}{2}\left|\alpha_{\nu}\right|^{2} s_{\nu}>0
$$

\section{Application}

In this section the proposed approach is applied to an academic coupled structure exhibiting a more refined dynamical behaviour. Particular properties of the power flow modes are also investigated and illustrated.

\subsection{Model description}

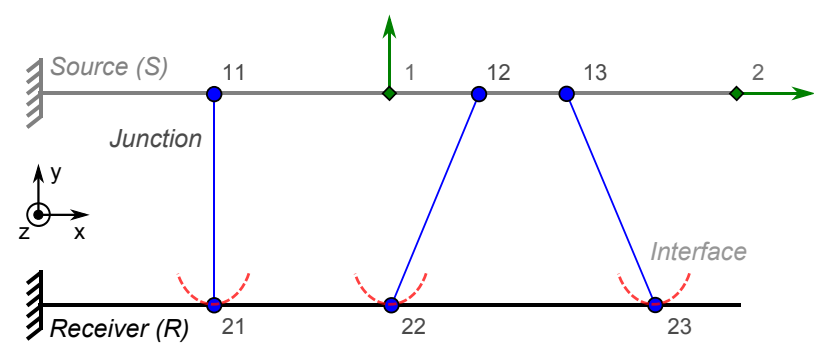

Figure 10: Coupled 2D-beam structure

The coupled structure depicted figure 10 consists in two clamped-free beams ${ }^{4}$ modeled using the finite element method, whose geometrical and material properties are given table 2 and which are similarly discretized. The problem is reduced to a plane one, coming down to $3 D O F s$ per node. A $10 \mathrm{~N}$ force is initially applied to the source substructure, respectively along $T_{y}$ and $T_{x}$ at node 1 and 2 (figure 10). This substructure is connected to the receiver one by three ball-joint links, modeled by local spring elements. The structure is globally submitted to a proportional damping. The considered frequency band between [0-2500 Hz] corresponds to the low frequencies (i.e. low modal overlap) of both substructures.

\subsection{Power flow modes}

According to the previously exposed formulation, power flow modes are determined by solving, at each frequency, the equivalent eigenproblem given equation (25). The interface is considered from the receiver side, consisting in constrained translational $D O F s$ at the interface nodes and leading to six power flow modes.

\footnotetext{
${ }^{4}$ The $x$-locations of nodes $1,2,11,12,13,21,22,23$ are $0.5,1,0.25,0.625,0.75,0.25,0.5,0.875$, respectively.
} 


\begin{tabular}{cccc}
\hline Structure & & Source & Receiver \\
\hline Dimensions & $(\mathrm{m})$ & $L=1, h=0,02$ \\
\hline Thickness & $(\mathrm{m})$ & 0,02 & 0,03 \\
\hline Young modulus & $(\mathrm{Pa})$ & $2,1.10^{11}$ & $6,7.10^{10}$ \\
\hline Density & $\left(\mathrm{kg} / \mathrm{m}^{3}\right)$ & 7800 & 2700 \\
\hline Damping & $($ proportional $)$ & $a=1.10^{-5}, b=1$ \\
\hline Junction stiffnesses & $(\mathrm{N} / \mathrm{m})$ & $k_{j}=1.10^{10}$ \\
\hline
\end{tabular}

Table 2: Initial parameters of the coupled 2D-beam structure

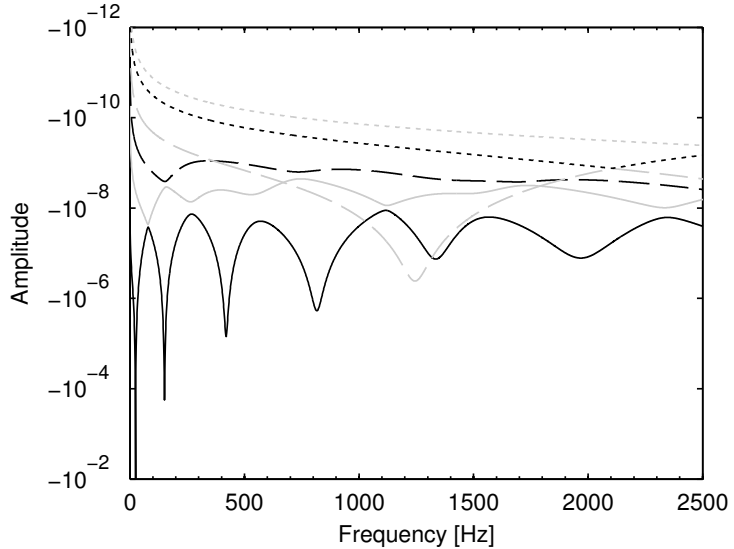

(a) Power flow eigenvalues:

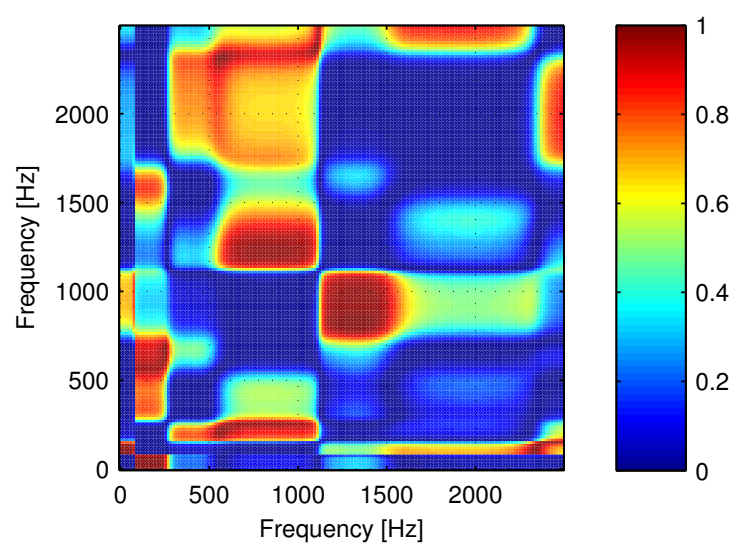

(b) Mode-mixing criterion between $\mathbf{g}_{1}$ and $\mathbf{g}_{2}$

Figure 11: Power flow modes of the coupled beam structure

Figure 11 shows the evolution of the power flow eigenvalues versus frequency. $s_{1}$ particularly shows a modal behaviour as it exhibits amplitude resonances at each normal vibration frequency of the receiver substructure ${ }^{5}$, excepted at $1250 \mathrm{~Hz}$ where the resonance occurs on $s_{4}$. This illustrates the decoupling between transverse and longitudinal vibrations of the beam: the first three eigenvalues are associated to its flexural behaviour while the next three are associated to its traction-compression one (whose first mode is located at $1250 \mathrm{~Hz}$ ).

Both crossing and veering of the eigenvalues can also be observed, especially between $s_{1}$ and $s_{2}$. Figure 11 (b) shows the visualization criterion, defined equation (18), applied to the associated eigenforces $\mathbf{g}_{1}$ and $\mathbf{g}_{2}$. According to the previous comments, mode-mixing phenomena can be seen at each antiresonance frequency, whose amplitude is conversely proportional to the distance between the eigenvalues: when these are very close the eigenvectors tend to totally exchange their deformed shapes (e.g. at $1100 \mathrm{~Hz}$ ).

\subsection{Dominant power flow paths at the interface}

The coupled structure is now subjected to the previously described loading. The resulting interface forces bewteen the receiver enf the junction are projected onto the power flow mode basis to derive, at each considered frequency,

\footnotetext{
${ }^{5}$ These natural frequencies are located at $25,150,420,815,1335,1970 \mathrm{~Hz}$ for the first flexural modes, and at $1250 \mathrm{~Hz}$ for the first tractioncompression one.
} 
the $\alpha_{v}$ coefficients defined equation (29). Considering their complex value and their participation in the transmitted power expression given equation (30), it is decided to focus on their absolute values.

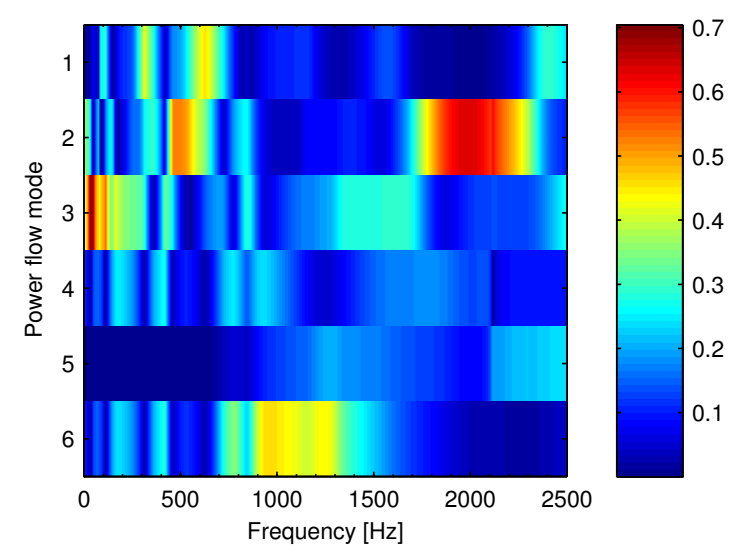

Figure 12: $\alpha_{v}$ projection coefficients at the interface of the coupled beam structure

Figure 12 shows these coefficients, which have been normalized so that their sum is equal to 1 at each frequency, representing the evolutions of the active power flow paths at the interface. For example, interface forces at $50 \mathrm{~Hz}$ are mostly collinear to $\mathbf{g}_{3}$ while they are distributed on both $\mathbf{g}_{1}$ and $\mathbf{g}_{2}$ at $600 \mathrm{~Hz}$. Once again the decoupling between the first three power flow modes, associated to the flexural behaviour, and the next three associated to longitudinal one can be observed. Moreover it can be noticed that the most active path is not necessarily related to the power flow eigenvalue having the higher absolute amplitude. This can be observed around $1200 \mathrm{~Hz}$ where the decomposition is about $45 \%$ along $\mathbf{g}_{6}$ against $20 \%$ along $\mathbf{g}_{5}$. Consequently, more power could be transmitted to the receiver substructure by considering different junction properties, another source substructure or different external loadings, in order to modify the dynamic behaviour of the structure.

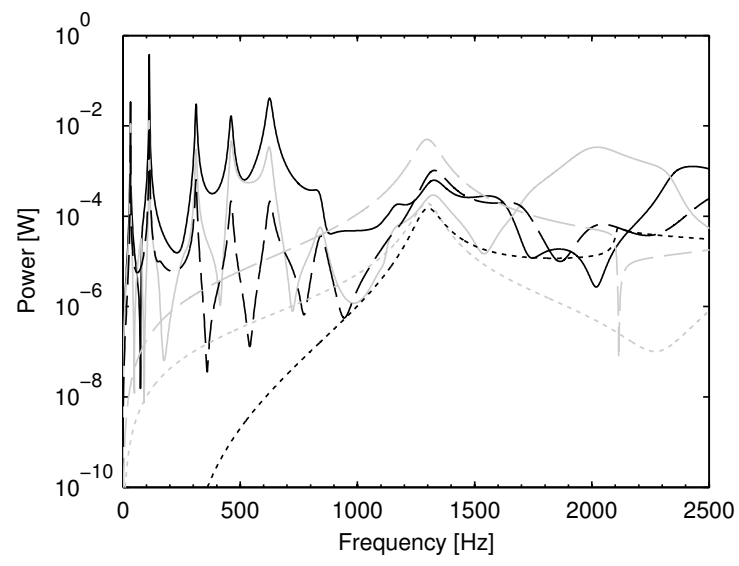

(a) Modal power flow:

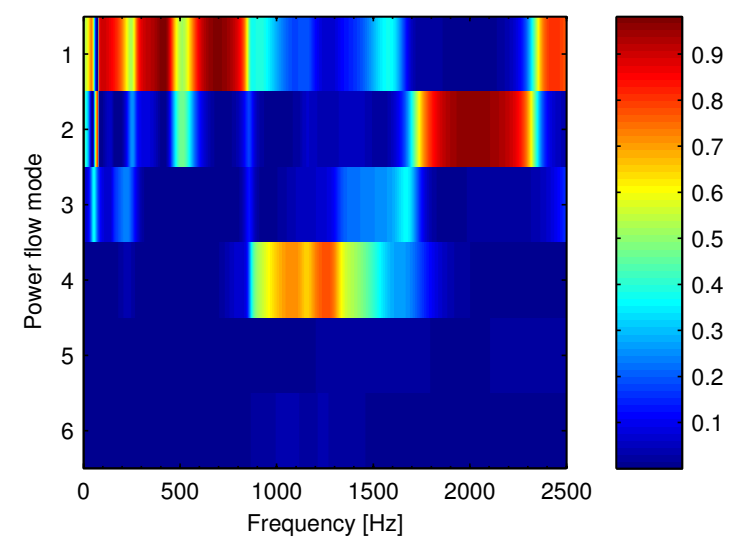

(b) Modal participation coefficients

$$
-P_{1},-P_{2},--P_{3},--P_{4}, \cdots P_{5}, \cdots P_{6}
$$

Figure 13: Modal power flows at the interface of the coupled beam structure

Figure 13 (a) shows the evolution of the modal power flows $P_{v}$ defined equation (31), which clearly depict the frequency behaviour of the coupled system. A normalized representation of these quantities is proposed figure 13 (b), 
to illustrate the distribution of the total transmitted power flow over the different power flow paths. In the same way as for the eigenvalues, the dominance of modes 1, 2 and 4 is emphasized: the directions of these eigenforces constitute a principal subspace of the power flow mode basis, through which most of the power is transmitted.

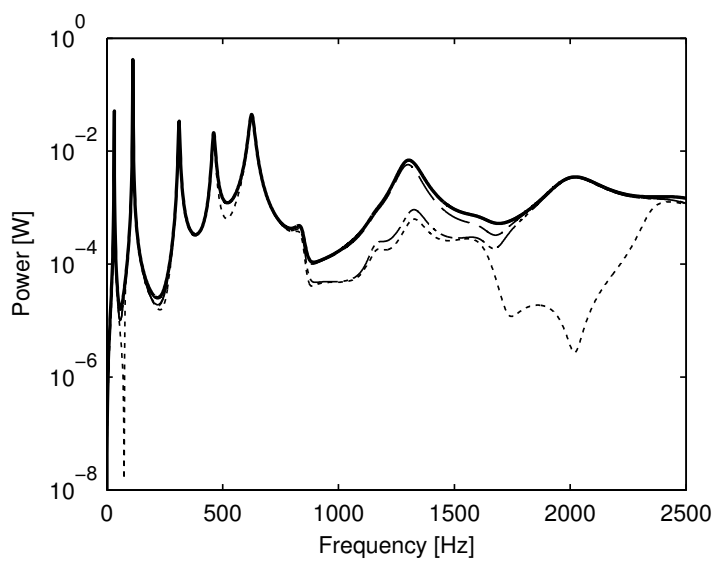

(a) Truncated modal power flows

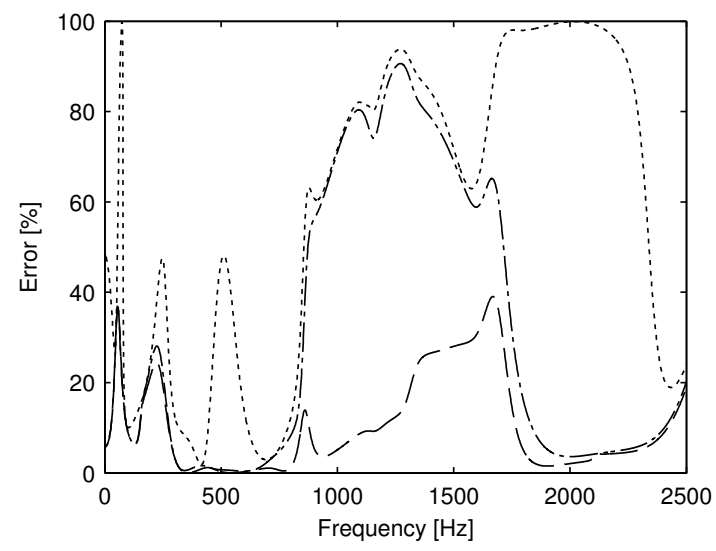

(b) Relative errors

Figure 14: Truncation effect on modal power flows of the coupled structure:

$$
-P_{j}^{t r}, \cdots P_{1},-\cdot-\left(P_{1}+P_{2}\right),--\left(P_{1}+P_{2}+P_{4}\right)
$$

Figure 14 (a) shows the evolution versus frequency of the transmitted power derived by truncating equation (30) to different subspaces, respectively consisting of $\mathbf{g}_{1},\left[\mathbf{g}_{1} \mathbf{g}_{2}\right]$ and $\left[\mathbf{g}_{1} \mathbf{g}_{2} \mathbf{g}_{4}\right.$ ]. Figure 14 (b) illustrates the associated relative errors with regard to the total transmitted power: in the last case, corresponding to the principal subspace, more than $60 \%$ of the power is released. Conversaly, the complementary susbspace concentrates less than $40 \%$ of the transmitted power, which constitutes an interesting property in a vibration isolation context.

Finally, it must be noticed that these results are quantatively related to the properties of the whole structure and the external loading (location, direction). Therefore, any modification of these will lead to new active paths and change the composition of the dominant subspace. However, it has been observed that this latter usually include one third of the power flow modes, representing about $60 \%$ of the transmitted power ${ }^{6}$.

\subsection{Combined force and moment at the interface}

Generally, interface forces between substructures shall apply simultaneously on both translational and rotational DOFs. However moments are often neglected as they are more difficult to measure experimentally. Detailed studies have therefore focused on rotational DOFs to identify situations where they must be taken into account [30]. Hence, the source descriptor method proposed by Petersson has emphasized their role in vibration transfers [31, 32, 33]. It has been shown that moments transmit more and more power flow as the frequency increases but also at low frequencies, when the junction is located near a structural discontinuity.

It is thus necessary to take account of all the DOFs involved at the interface in the analysis of the coupled structure. However, combined force and moment excitations are not directly comparable due to their different dimensions, hence the interest in considering the associated power flows. To overcome this difficulty, Moorhouse has introduced a dimensionless matrix formulation based on a mobility approach, normalizing cross-mobility terms by both force and moment point-mobility ones [34]. It is therefore possible to quantify the coupling between different excitations and to determine the extent to which an applied force or moment at a given location will affect the power transmitted by another force or moment.

\footnotetext{
${ }^{6}$ This property of quadratic energy operators has already been highlighted in the literature, e.g. by Soize ??.
} 
Similarly, so as not to favor some directions over others in power flow modes, this weighting procedure is applied using the dimensionless diagonal matrix $\mathbf{D} \in \mathbb{R}^{\left(N_{j}, N_{j}\right)}$ defined by ${ }^{7}$

$$
\mathbf{D}_{(i, i)}=\frac{1}{\sqrt{\mid \Gamma^{\mathfrak{J}}\left({ }_{(i, i)} \mid\right.}}
$$

The weighted quantities $\overline{\boldsymbol{\Gamma}}^{\mathfrak{I}} \in \mathbb{R}^{\left(N_{j}, N_{j}\right)}$ and $\overline{\mathbf{f}}_{j} \in \mathbb{C}^{\left(N_{j}, 1\right)}$ are then obtained as follows

$$
\begin{aligned}
\overline{\boldsymbol{\Gamma}}^{\mathfrak{I}} & =\mathbf{D} \boldsymbol{\Gamma}^{\mathfrak{I}} \mathbf{D} \\
\overline{\mathbf{f}}_{j} & =\mathbf{D}^{-1} \mathbf{f}_{j}
\end{aligned}
$$

allowing the expression of the power transmitted at the interface to be rewritten

$$
P_{j}^{t r}\left(\omega_{0}\right)=-\frac{\omega_{0}}{2} \mathbf{f}_{j}^{H} \boldsymbol{\Gamma}^{\mathfrak{J}} \mathbf{f}_{j}=-\frac{\omega_{0}}{2} \overline{\mathbf{f}}_{j}^{H} \overline{\boldsymbol{\Gamma}}^{\mathfrak{J}} \overline{\mathbf{f}}_{j}
$$

The matrix $\overline{\boldsymbol{\Gamma}}^{\mathfrak{I}}$ having the same properties as $\boldsymbol{\Gamma}^{\mathfrak{J}}$, all the previously detailed approach can be applied to determine normalized power flow modes.

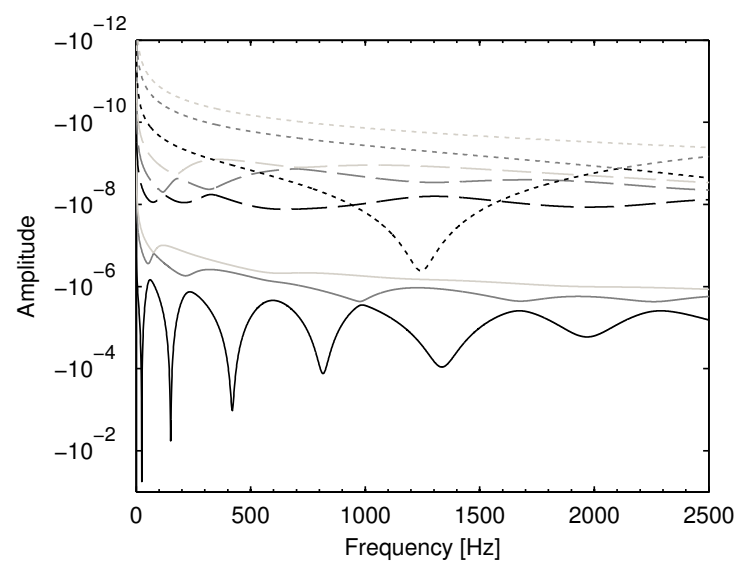

(a) Before weighting

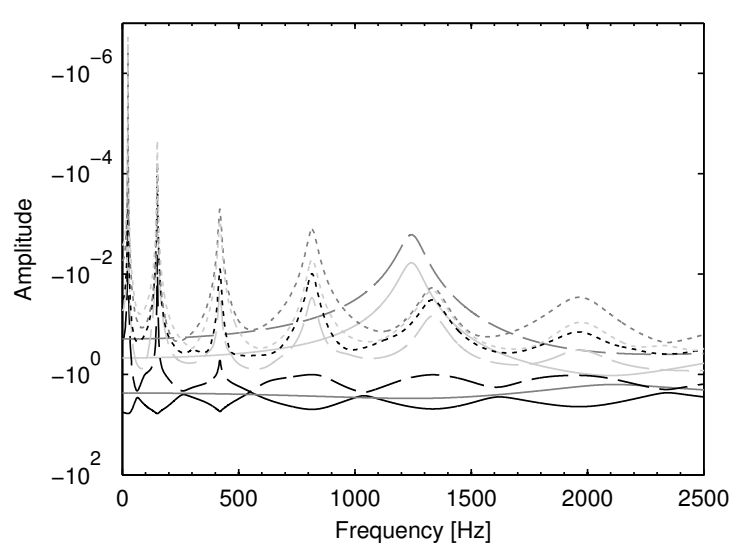

(b) After weighting

Figure 15: Generalized power flow eigenvalues:

$$
-s_{1},-s_{2},-s_{3},--s_{4},--s_{5},--s_{6}, \cdots s_{7}, \cdots s_{8}, \cdots s_{9}
$$

Figure 15 compares the power flow eigenvalues derived from the initial and dimensionless normalized formulations, when all the interface DOFs of the coupled beam structure are constrained. The initial behaviour is close to the previously described one (figure 11 (a)) but with higher absolute amplitudes, which confirms the stiffening of the interface constraints ${ }^{8}$. While globally different, the normalized behaviour denotes the same properties: the eigenvalues remain negative, they exhibit amplitude resonances and a principal subspace can be observed (the associated eigenvalues having smaller antiresonances).

The evolution of the power flow modal participation coefficients is illustrated figure 16. Once again the prominent role of the principal subspace can be observed, although it may not consists of the same eigenforces before and

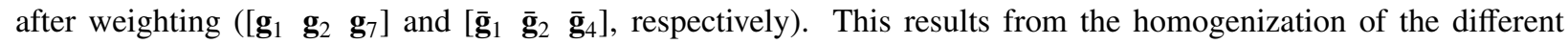
DOFs components after weighting, which generates spatially more complicated paths. However, the power flow path associated to the first longitudinal vibration mode remains decoupled and also becomes more important $\left(\mathbf{g}_{7} \rightarrow \overline{\mathbf{g}}_{2}\right)$.

\footnotetext{
${ }^{7}$ According to Ji et al. various weighting matrices can be used, based on different matrix norms [35].

${ }^{8}$ The flexural behaviour is highly impacted by the rotational stiffening, contrary to the traction-compression one whose first mode remains unaffected at $1250 \mathrm{~Hz}$.
} 


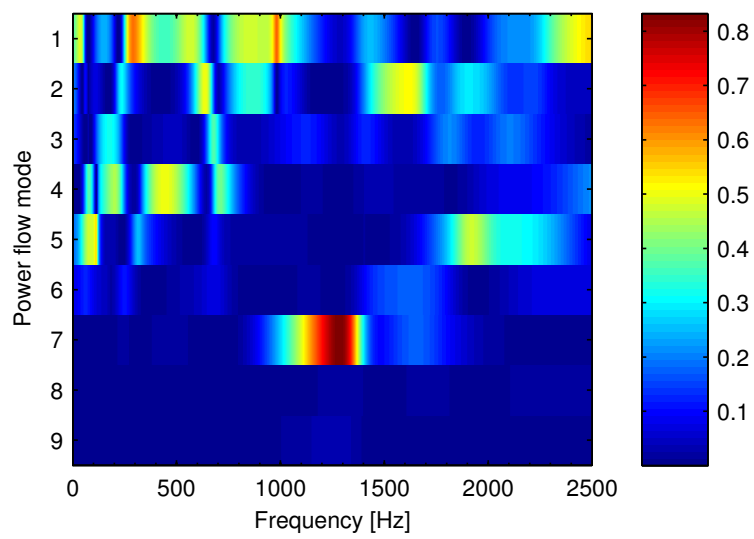

(a) Before weighting

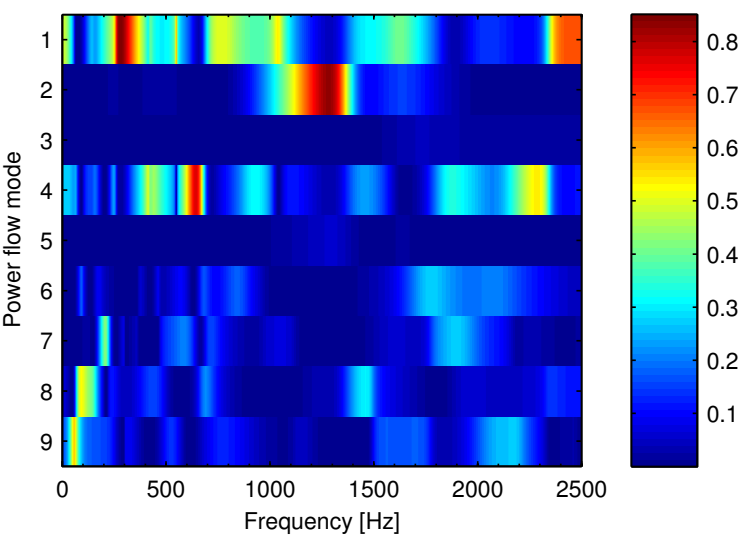

(b) After weighting

Figure 16: Power flow modal participation coefficients

\subsection{Extension to mid-frequency}

It has been exposed that the proposed power flow mode method strongly depends on the frequency, implying mode-mixing phenomena of the eigenforces and modifying the dominant power flow paths. However it has been observed that their variations slow down at power flow resonances, where the dynamic behaviour is controlled by a single normal vibration mode (unlike antiresonances). Moreover this variation tends to stabilize as the considered frequency gets closer to the high frequency band associated to the receiver substructure (i.e. high modal overlap). Hence, it seems interesting to determine a single, frequency-independent, power flow mode basis to characterize the interface forces on a defined frequency band.

Two approaches have been used to determine a reference power flow mode basis. The first one has been proposed by Cazzolato and Hansen in the context of vibroacoustic active isolation to filter radiation modes [36]. Adapted to our objective, this consists in projecting each power flow basis $\mathbf{G}$ derived over a given frequency band onto the one associated to a single reference frequency $\mathbf{G}_{r e f}$, by determining several orthogonal projection matrices $\mathbf{P}$

$$
\mathbf{P}=\mathbf{G}_{r e f}^{-1} \mathbf{G}=\mathbf{G}_{r e f}^{T} \mathbf{G}
$$

The expression of the imaginary part of the dynamic flexibility matrix at the interface given equation (27) can thus be rewritten as

$$
\boldsymbol{\Gamma}^{\mathfrak{J}}=\mathbf{G S G}^{T}=\left(\mathbf{G}_{r e f} \mathbf{G}_{r e f}^{T}\right) \mathbf{G S G}^{T}\left(\mathbf{G}_{r e f} \mathbf{G}_{r e f}^{T}\right)=\mathbf{G}_{r e f} \mathbf{S}_{r e f} \mathbf{G}_{r e f}^{T}
$$

where $\mathbf{S}_{r e f}=\mathbf{P S P} \mathbf{P}^{T} \in \mathbb{R}^{\left(N_{j}, N_{j}\right)}$ is a diagonally dominant matrix, excepted at the reference frequency where it represents the spectral matrix of the power flow eigenvalues. It is further filtered to obtain independent relations in the power flow mode space by imposing

$$
\tilde{\mathbf{S}}_{r e f}=\operatorname{diag}\left(\mathbf{S}_{r e f}\right)=\operatorname{diag}\left(\mathbf{P S P}^{T}\right)
$$

The transmitted power flow is finally expressed according to the reference basis and this frequency-dependent filtered matrix

$$
P_{j}^{t r}\left(\omega_{0}\right) \approx-\frac{\omega_{0}}{2} \mathbf{f}_{j}^{H} \mathbf{G}_{r e f} \tilde{\mathbf{S}}_{r e f} \mathbf{G}_{r e f}^{T} \mathbf{f}_{j}
$$

However, the main drawback remains the need to first determine all the power flow modes at each frequency: an $a$ priori procedure based on the average behaviour of the source substructure would be computationally more appealing. Therefore, the concept of average dynamic flexibility matrix has been introduced

$$
\boldsymbol{\Gamma}_{a v}^{\mathfrak{J}}=\frac{1}{N} \sum_{i=1}^{N} \boldsymbol{\Gamma}^{\mathfrak{J}}\left(\omega_{i}\right)
$$


allowing to solve only once the equivalent eigenproblem to determine the power flow mode on a given frequency band (cut into $N$ frequency steps). This finally leads to the following approximation of the transmitted power flow

$$
P_{j}^{t r}(\omega) \approx-\frac{\omega_{0}}{2} \mathbf{f}_{j}^{H} \mathbf{G}_{\text {moy }} \mathbf{S}_{\text {moy }} \mathbf{G}_{\text {moy }}^{T} \mathbf{f}_{j}
$$

While this approach seems barely credible at low frequencies for a purely modal dynamic behaviour, it shares similarities with some mid-frequency model reduction methods, as developed by Soize et al. [37, 38].

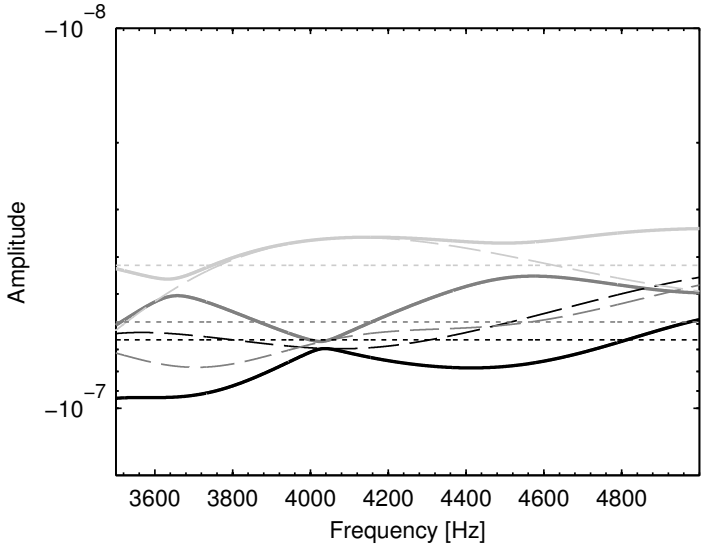

(a) Power flow eigenvalues: $-s_{1},-s_{2},-s_{3}$

- exact, -- normalized at $4040 \mathrm{~Hz}$, -. averaged

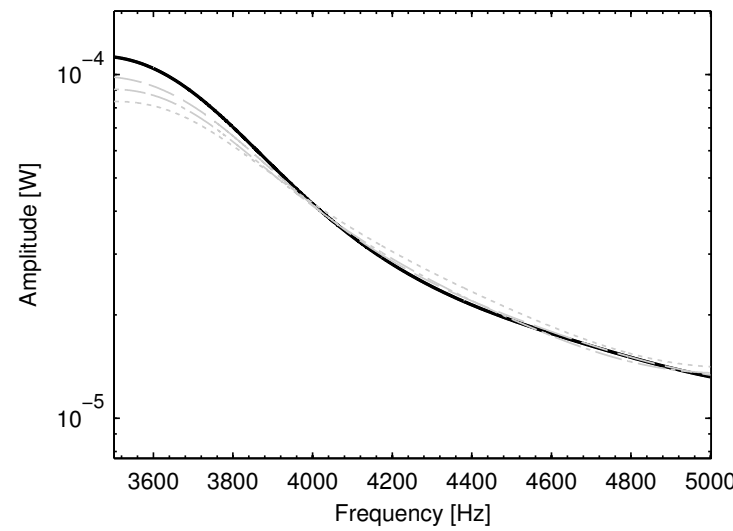

(b) Transmitted power: - exact, - normalized eigenforces at $4040 \mathrm{~Hz}$, fixed eigenforces at $4040 \mathrm{~Hz}, \cdots$ averaged

Figure 17: Mid-frequency power flow approximations

To illustrate both approaches, the physical parameters of the coupled beam structure have been modified to depict a global mid-frequency behaviour considering the dynamic behaviour of each substructure: the source and receiver have been separately tuned to present a low and a high modal overlap, respectively, on the considered frequency band. Figure 17 (a) shows the derived power flow eigenvalues on 3500-5000 Hz. Both procedures give results of the same order of magnitude, even if the normalized one $\left(f_{\text {ref }}=4040 \mathrm{~Hz}\right)$ introduces small variations due to the low modemixing of the eigenforces. Figure 17 (b) represents the synthesized transmitted power flows, exhibiting little relative errors. A variant of the normalization procedure is also proposed, which consists in not updating the eigenvalue matrix and keeping it constantly equal to the power flow eigenvalues at the reference frequency. The obtained estimates seem to give better results than the average flexibility ones.

\section{Conclusion}

A power flow mode method has been presented, based on a displacement formulation of the average power. It has been demonstrated that minimizing this quantity with regard to the external applied forces amounts to solving an equivalent eigenvalue problem associated to the imaginary part of the dynamic flexibility matrix of the structure. The derived eigenvalues and eigenforces provide quantitative and qualitative information on the power flowing inside the structure, respectively. A parametric study has emphasized the complex frequency-dependent behaviour of these quantities with regard to the parameters of the system.

This approach has been further applied to study the power transmitted between a source substructure, submitted to a given external loading, and a receiver substructure. It has been noticed that power flow modes only depend on the receiver properties. Projection coefficients have been derived which allow characterizing interface forces, especially by identifying the directions constituting the different power flow paths. Is has been noticed that some of them constitute a dominant subspace through which most of the power flow is transmitted. 
The role of moments in power transmission between different substructures has also been underlined. However, the involved behaviours remain rather complex and difficult to expose. While the proposed weighting procedure is physically justified and easy to implement, it complicates the determination of the dominant power flow paths, whose number increases as the vibrational behaviour of the substructures gets more complex. Moreover, considering that mode-mixing phenomena of the eigenforces slow down as the frequency tends toward the high-frequency band of the receiver substructure, two procedures have been presented to derive a single fixed power flow mode basis. While computationally cost effective, it could be interesting to compare the derived results to SEA-like ones, which are based on low-frequency approaches to derive energy flow coefficients representing modal couplings between substructures $[39,40]$.

Finally, considering the useful insights provided by this study on the vibration transfer mechanisms between components of assembled structures, a robust design approach of structural interfaces has been proposed by the authors in an upcoming paper. Optimization procedures have been implemented and compared to minimize the power transmitted between a source and a receiver substructure, with respect to the interface physical parameters, leading to promising results.

\section{Acknowledgments}

This work has been funded by the Future Preparation - Research and Technology Sub-Directorate of the Centre National d'Études Spatiales (CNES) - Launchers Directorate (Paris, France).

\section{References}

[1] P. Gardonio, S. Elliott, R. Pinnington, Active isolation of structural vibration on a multiple-degree-of-freedom system, part ii: effectiveness of active control strategies, Journal of Sound and Vibration 207 (1997) 95- 121.

[2] C. Beijers, A modeling approach to hybrid isolation of structure-borne sound, Ph.D. thesis, University of Twente, Pays-Bas, 2005.

[3] L. Cremer, M. Heckl, B. Petersson, Structure-borne sound, 3e edition ed., Springer Verlag, Berlin, Allemagne, 2005.

[4] M. Brennan, N. Ferguson, Advanced applications in acoustics, noise and vibration, Spon Press, Londres, 2004 , pp. 530- 580.

[5] P. Gardonio, S. Elliott, R. J. Pinnington, Active isolation of structural vibration on a multiple-degree-of-freedom system, part i: the dynamics of the system, Journal of Sound and Vibration 207 (1997) $61-93$.

[6] R. Lyon, G. Maidanik, Power flow between linearly coupled oscillators, Journal of the Acoustical Society of America 34 (1962) 623 - 639.

[7] H. Goyder, R. White, Vibrational power flow from machines into built-up structures, part i: Introduction and approximate analyses of beam and plate-like foundations, Journal of Sound and Vibration 68 (1980) $59-75$.

[8] H. Goyder, R. White, Vibrational power flow from machines into built-up structures, part ii: Wave propagation and power flow in beamstiffened plates, Journal of Sound and Vibration 68 (1980) $77-96$

[9] H. Goyder, R. White, Vibrational power flow from machines into built-up structures, part iii: Power flow through isolation systems, Journal of Sound and Vibration 68 (1980) $97-117$.

[10] R. Pinnington, R. White, Power flow through machine isolators to resonant and non-resonant beams, Journal of Sound and Vibration 75 (1981) $179-197$

[11] R. Pinnington, Using the envelope of the frequency response in the measurement of power absorbed by a finite structure, Journal of Sound and Vibration 109 (1986) 127 - 139.

[12] P. Gardonio, M. Brennan, On the origins and developments of mobility and impedance methods in structural dynamics, Journal of Sound and Vibration 249 (2002) $557-573$

[13] B. Petersson, J. Plunt, On effective mobilities in the prediction of structure-borne sound transmission between a source structure and a receiving structure, part i: Theoretical background and basic experimental studies, Journal of Sound and Vibration 82 (1982) 517 - 529.

[14] J. Cuschieri, Vibration transmission through periodic structures using a mobility power flow approach, Journal of Sound and Vibration 143 (1990) $65-74$.

[15] J. Mondot, B. Petersson, Characterization of structure-borne sound sources: The source descriptor and the coupling function, Journal of Sound and Vibration 114 (1987) 507 - 518.

[16] R. Fulford, B. Gibbs, Structure-borne sound power and source characterization in multi-point-connected systems, part i: case studies for assumed force distributions, Journal of Sound and Vibration 204 (1997) $659-677$.

[17] H. Bonhoff, B. Petersson, The influence of cross-order terms in interface mobilities for structure-borne sound source characterization: Forceorder distribution, Journal of Sound and Vibration 322 (2009) 241 - 254.

[18] H. Bonhoff, B. Petersson, The influence of cross-order terms in interface mobilities for structure-borne sound source characterization, Journal of Sound and Vibration 329 (2010) $3280-3303$.

[19] D. Miller, S. Hall, A. von Flotow, Optimal control of power flow at structural junctions, Journal of Sound and Vibration 140 (1990) 475 497.

[20] J. Su, A. Moorhouse, B. Gibbs, Towards a practical characterization for structure-borne sound sources based on mobility techniques, Journal of Sound and Vibration 185 (1995) $737-741$. 
[21] Y. Xiong, J. Xing, W. Price, A power flow mode theory based on a system's damping distribution and power flow design approaches, Proceedings of the Royal Society A: Mathematical, Physical and Engineering Science 461 (2005) 3381 - 3411.

[22] F. Bessac, L. Gagliardini, J.-L. Guyader, Coupling eigenvalues and eigenvectors: a tool for investigating the vibroacoustic behaviour of coupled vibrating systems, Journal of Sound and Vibration 191 (1996) $881-899$.

[23] T. Weisser, Caractérisation dynamique et conception robuste d'interfaces de structures, Ph.D. thesis, Université de Franche-Comté, Besançon, 2012.

[24] Y. Bobrovnitskii, Some energy relations for mechanical systems, in: IUTAM Symposium on Statistical Energy Analysis, Southampton, Royaume-Uni, 1997.

[25] L.-O. Gonidou, Dynamic characterization of structural interfaces, Proceedings of the the Spacecraft and Launch Vehicle Dynamic Environments Workshop (2007).

[26] P. Lancaster, M. Tismenetsky, The theory of matrices, Academic Press, 1985.

[27] L. Ratsifandrihana, Amélioration des procédures d'identification modale des structures par appropriation automatique et utilisation de forces non controlées, Ph.D. thesis, Université de Franche-Comté, Besançon, 1995.

[28] C. Pierre, Mode localization and eigenvalue loci veering phenomena in disordered structures, Journal of Sound and Vibration 126 (1988) 485 -502 .

[29] X. Liu, Behavior of derivatives of eigenvalues and eigenvectors in curve veering and mode localization and their relation to close eigenvalues, Journal of Sound and Vibration 256 (2002) $551-564$.

[30] R. Fulford, B. Petersson, The role of moments on the vibration transmission in built-up structures, Journal of Sound and Vibration 227 (1999) $479-510$.

[31] B. Petersson, Structural acoustic power transmission by point moment and force excitation, part i: beam- and frame-like structures, Journal of Sound and Vibration 160 (1993) $43-66$.

[32] B. Petersson, Structural acoustic power transmission by point moment and force excitation, part ii: plate-like structures, Journal of Sound and Vibration 160 (1993) $67-91$.

[33] B. Petersson, B. Gibbs, Use of the source descriptor concept in studies of multi-point and multi-directional vibrational sources, Journal of Sound and Vibration 168 (1993) 157 - 176.

[34] A. Moorhouse, A dimensionless mobility formulation for evaluation of force and moment excitation of structures, Journal of the Acoustical Society of America 112 (2002) $972-980$

[35] L. Ji, B. Mace, R. Pinnington, A power mode approach to estimating vibrational power transmitted by multiple sources, Journal of Sound and Vibration 265 (2003) $387-399$.

[36] B. Cazzolato, C. Hansen, Active control of sound transmission using structural error sensing, Journal of the Acoustical Society of America 104 (1998) $2878-2889$.

[37] R. Ohayon, C. Soize, Structural acoustics and vibration, Academic Press, Londres, Royaume-Uni, 1998.

[38] C. Soize, Reduced models in the medium frequency range for general dissipative structural-dynamics systems, European Journal of Mechanics - A/Solids 17 (1998) 657 - 685.

[39] C. Fred, A sea-like approach for the derivation of energy flow coefficients with a finite element model, Journal of Sound and Vibration 199 (1997) $645-666$

[40] B. Mace, Statistical energy analysis: coupling loss factors, indirect coupling and system modes, Journal of Sound and Vibration 279 (2005) $141-170$. 